\title{
PREreview of "Doc2b Ca2+-binding site mutants act as a gain of function at rest and loss of function during neuronal activity"
}

\author{
Yufei $\mathrm{Si}^{1}$ \\ ${ }^{1}$ University of California, Santa Cruz
}

April 28, 2020

This is a preprint review of Doc2 b $\mathbf{C a}^{2+}$-binding site mutants act as a gain of function at rest and loss of function during neuronal activity by Quentin Bourgeois-Jaarsma, Matthijs Verhage and Alexander J Groffen. The preprint was originally posted on bioRxiv on January 31, 2019 (DOI: https: //doi.org/10.1101/536581).

In this manuscript, Bourgeois-Jaarsma and colleagues work on identifying the role of the Ca2+-binding double $\mathrm{C} 2$ protein, Doc2b, in neurotransmission. They focus on two Doc2b mutants (Doc2bDN and Doc2b6A) and observe that the two mutant proteins 1) localize at plasma membrane of neurons less activityindependently than wildtype Doc2b, and 2) show different Ca2+-dependent pattern from wildtype Doc2b when binding with phospholipid in vitro. They also show that in cultured neurons (either wild type or Doc-KO), overexpression of the two mutants 3) both increases the frequency of spontaneous neurotransmitter release at rest, 4) introduces different patterns of response overtime when repetitively stimulated, but 5) doesn't affect the neurons' morphology. From these, they conclude that the two mutants share similar behaviors both at rest and during neuronal activity: increased spontaneous activity at rest and decreased activity during neuronal activity, and thus showing Doc2b plays an important role in Ca2+-dependent phospholipid association.

In general, the logic of this study is clear, and the authors present their work in a way easy to follow. After visualizing subcellular localization of the mutants, the authors then test their Ca2+-dependency in phospholipid binding, effects of overexpression of them in neurons at rest, during activity and on morphology. Also, different models, strategies and experiments are used to address questions and hypothesis at each step. The combination of in vitro Ca2+ binding experiment, in vivo subcellular localization and physiology recording really makes the story coherent and intriguing to think deeply about. Moreover, the authors consider various possibilities and carefully design their controls.

However, one of the weaknesses would be the conclusion that overexpression of the mutant Doc $2 \mathrm{~b}$ proteins would lead to an increased short-term depression. The authors somehow tend to over-normalize the data in some cases and make the interpretations not convincing enough.

\section{Detailed comments:}

\section{Significance}

There are some controversies in the field that the authors want to address, and the authors claim to have provided "a unifying explanation for seemingly conflicting data and emphasize the importance of the Ca2+dependent phospholipid association in Doc2b-mediated secretory regulation": 
1) The gain- or loss-of-function debate of the Doc2b mutant proteins (Doc2bDN and Doc2b6A were reported differently in previous studies). The authors suggest that they have reconciled the previous studies by showing DN and 6A mutants do not have different gain- or loss-of-function pattern but show similar behavior: increased activity (gain-of-function) at rest but impaired activity at high [Ca2+] (loss-of-function) during neuronal activity.

2) Whether the Doc2b proteins function as $\mathrm{Ca} 2+$ sensors. The authors believe Doc2b functions as a Ca2+ sensor and argue that "the strict correlation between plasma membrane association and spontaneous release frequency supports a direct role as a $\mathrm{Ca} 2+$ sensor. In addition, a $\mathrm{Ca} 2+-$ dependent function in synaptic recovery is also supported by the data".

\section{Observation}

There are some concerns about their observation:

The authors use normalization for most of the data they observe, and it can help visualize trends of data thus aiding our understanding. However, it can also blind us from seeing the data if not normalized in a right way. For example:

A major concern is that in figure 5 and figure 8, absolute and normalized total charge (E\&F, L\&M in figure5; $\mathrm{D} \& \mathrm{E}, \mathrm{J} \& \mathrm{~K}$ in figure 8) show much different trends, and the authors claim to see a short-term depression based on the normalized trends. It seems, from the figure, that the authors normalized that data with the peak value in each group (it is not written in the manuscript), but one can argue that there difference between mutant and control groups are actually the peak value, possibly making it a biased standard to be normalized. For instance, what we see in figure $5 \mathrm{E}$, the absolute charge, is heavily overlapping trends after the initial peaks for each protein. One can conclude from here that the Doc $2 \mathrm{~b}$ mutants affect the initial amount of charge while but not later when repetitively stimulated. But after the normalization, the initial peaks are normalized, and from the later trends the authors conclude that Doc $2 \mathrm{~b}$ mutants lead to a fast depression of EPSC charge. The author reason that the observation after normalization suggests that "the larger initial EPSC charge in mutant expressing neurons contributes to the phenotype", but the question is whether the phenotype really is the depression or just the large initial peak.

Another similar concern is that in figure 5 and 8 , the authors measure recovery as the normalized response charge after repetitive stimulation and a short rest. There's nowhere in the manuscript mentioning how exactly the normalization is done, and there's no corresponding absolute data available to refer to. Note that normalization is not used in for single evoked EPSCs (figure 5 (A-C), figure 8 (A-C)), which makes it a little harder for readers to compare with other data known already and follow better.

A minor concern would be that in Figure 2 the authors only present the normalized eGFP/mCherry intensity in each case (presumably normalized by the peak intensity in naïve and stimulation conditions). Without absolute fluorescence intensity, we can only see the relative differences between naïve and stimulation scenarios, but not the specific change of protein distributions between resting state and stimulated state of neurons.

Another minor concern is that in figure $3 \mathrm{H}$ and $\mathrm{P}$, again normalized curves are plotted. But from the same figure $\mathrm{E}-\mathrm{G}$ and $\mathrm{M}-\mathrm{O}$, we can see that mutant $\mathrm{C} 2 \mathrm{ABs}$ cannot get to as large maximal values as the WT ones. The authors see the differences in lipid binding increase with higher $\mathrm{Ca} 2+$ concentration, yet there is also an impaired lipid binding ability for mutant proteins worth noticing.

\section{Interpretation}


The authors support their inferences well with detailed and logically conveyed observations. The only thing I am not totally convinced is the conclusion of overexpression of Doc2bDN and Doc2b6A mutants results in short-term depression after repetitive stimulation. It might be helpful to show the absolute data rather than only normalized ones for the "recovery" measurements in figure 5 and 8 . Also, if the authors can give some more justifications of the data showing short-term depression rather than larger initial peak.

\section{Clarity}

The manuscript is generally easy to read and free of jargon, typos and grammatical or conceptual errors. The authors are doing an especially good job in distinguishing similar but different concepts throughout the manuscript. The information provided in figures and legends are clear and accurate, especially accessible to non-specialist to follow.

There are just some minor concerns about figure labeling:

Figure 3 (M-P). there is a dash line in each panel with "rest" and "activated" on each side. But there's nowhere else in the manuscript explaining how that is defined.

Figure 5 and 8, "paired pulse ratio" is used as one of the measurements, and it might be basic concept for people in the field, but with no explanation about it in the manuscript, it is hard for non-expert readers to catch up.

Details about EPSC experiments. One concern is that although it might be very basic knowledge on neurotransmitter release, it's probably still better if EPSC represents neurotransmitter release is explained somewhere to help people not familiar with the topic to follow.

Details about data analysis. It might be better if the authors could mention more about how the data are analyzed, so that people not familiar with the topic and their methods can also follow better. 